\title{
Relaciones entre autoconcepto académico, atribuciones de éxito y fracaso, y rendimiento académico en escolares preadolescentes
}

\author{
Giovanna Moreano ${ }^{1}$
}

\begin{abstract}
La presente investigación busca identificar las relaciones que se tienden entre las variables mencionadas. 284 estudiantes de $5^{\circ}$ y $6^{\circ}$ grado de primaria conformaron la muestra. Para medir las variables se aplicó el Cuestionario de Autodescripción (SDQ-1) y la Escala de Atribuciones de Sydney (SAS). Asimismo, se recogieron los promedios finales de las áreas de matemáticas y comunicación integral como indicadores de rendimiento. Un análisis factorial exploratorio del SAS mostró cinco factores, tres de los cuales demostraban que los estudiantes no discriminaban entre esfuerzo y habilidad. Sobre el SDQ, los resultados confirmaron que los niños diferenciaban entre las diferentes dimensiones del autoconcepto. En general, los participantes mostraron un autoconcepto favorable en todas sus dimensiones, lo cual estaría relacionado con el marcado locus de control interno que mostraron. Asimismo, pudo apreciarse la tendencia notable a aceptar el éxito por causas internas y negar las posibilidades de fracaso.

Palabras clave: autoconcepto académico, atribuciones de éxito y fracaso, rendimiento académico.
\end{abstract}

Relationship between academic self-concept, causal attribution for success and failure, and academic achievement in pre-adolescents

This study shows the relationship between the variables mentioned above in 284 primary school students in Lima ( $5^{\text {th }}$ and $6^{\text {th }}$ grades). The Self Description Questionnaire (SDQ-1) and the Sydney Attribution Scale (SAS) were applied with a previous validation process. Mathematics and Language final grades were assumed as academic achievement indicators. SAS exploratory factorial analysis showed five factors, three of them demonstrated that children do not discriminate between effort and ability. About SDQ, results confirmed that children can differentiate between self-concept dimensions. Participants showed a favorable self-concept in all dimensions and a strong internal locus of control. In the same way, there was a tendency for students to accept success for internal causes and totally deny failure.

Keywords: academic self-concept, causal attribution for success and failure, academic achievement.

1 Licenciada en Psicología Educacional por la PUCP. Consultora del Equipo de Análisis de la Unidad de Medición de la Calidad del Ministerio de Educación. Jefa de Práctica en cursos de formación básica de la especialidad de Psicología. Temas de investigación: variables psicológicas del docente y de los alumnos relacionadas con el rendimiento académico estudiantil. Correo electrónico: moreano.g@pucp.edu.pe 

El ingreso del niño a la escuela y su permanencia en ella se convierte en una experiencia importante pues favorece el desarrollo de las dimensiones cognitiva, social, emocional y física. Sin embargo, en la escuela existe un énfasis en el desarrollo de la dimensión cognitiva en desmedro de las otras dimensiones. Más aún, se pretende que los estudiantes logren desarrollar las competencias esperadas y el consiguiente rendimiento académico positivo trabajando sólo a nivel cognitivo sin considerar que lo social y emocional pueden favorecer o retrasar esta meta. La literatura actual provee información sobre cómo el desarrollo de las dimensiones emocional y social aporta al desarrollo de habilidades y competencias de modo que puedan lograrse ambos, el rendimiento académico positivo y el bienestar psicológico del estudiante. De este modo, en el aspecto emocional y motivacional, se considera a las imágenes y percepciones que los estudiantes tienen sobre sí mismos como influyentes en el rendimiento académico. Asimismo, el docente se constituye en una persona clave para la formación de imágenes y autopercepciones positivas pues sus observaciones, críticas y reforzamientos pueden llevar a un estudiante a persistir en una tarea o a evitarla por miedo a fracasar. En este contexto se hace necesario concientizar a los profesores del importante rol que cumplen como formadores de un autoconcepto favorable en sus estudiantes, así como de cogniciones, en general, que lleven al estudiante a mostrar motivación y confianza en sus propias capacidades.

Actualmente y pese a la importancia planteada, nuestro medio carece de estudios que traten de explorar las relaciones entre variables motivacionales como el autoconcepto académico y las 
atribuciones de éxito y fracaso con el rendimiento académico en estudiantes de nivel primario en escuelas estatales. Esta investigación pretende cubrir esa carencia esperando que los resultados guíen la elaboración de programas de intervención que ayuden al niño a formarse un sentido de competencia y así tenga mayores posibilidades de lograr éxito frente a una tarea.

Previamente a presentar los resultados de la investigación, se revisará brevemente el estado de arte sobre las variables de estudio en cuestión y los aspectos metodológicos considerados en el estudio.

\section{Autoconcepto académico}

Existen muchas formas de definir el autoconcepto, sin embargo, el actual estado de arte respecto del tema considera algunos puntos de acuerdo. Uno de ellos es su carácter multidimensional. En esta línea, Harter define el autoconcepto como la percepción que el individuo tiene de sí a partir de los diferentes aspectos de su self (Bong \& Skaalvic, 2003; Harter, 1999). En correspondencia con esta definición, Shavelson, Hubner y Stanton (como se cita en Marsh, 1990) desarrollaron un modelo multifacético y jerárquico del autoconcepto que considera la existencia de dos dimensiones del self: la académica y la no académica. La dimensión académica incluye el autoconcepto en tres dominios específicos: matemática, lectura y académico general. La dimensión no académica, por su parte, incluye el autoconcepto relacionado con cuatro aspectos: apariencia física, relación con padres, habilidad física y relación con pares. Otro aspecto que define al autoconcepto es el rol de las interacciones sociales y sus influencias en la formación del autoconcepto. Las personas que forman parte del ambiente de un individuo le proveen información en la forma de evaluaciones, 
reforzamientos, etc., los cuales interpretará y asimilará en su autoconcepto (Marsh, 1990). Finalmente se tiene una visión del self como sistema, no sólo a un nivel cognitivo, sino también afectivo que media la organización de la experiencia del individuo y la regulación de sus conductas.

Todas estas características pueden ser observadas a lo largo de las diferentes etapas de desarrollo. Durante la infancia el niño se muestra capaz de organizar el conjunto de percepciones sobre sí mismo conforme va avanzando en su proceso de desarrollo (Bouffard, Marcoux, Vezeau \& Bordeleau, 2003; Saura, 1995). Así, luego de atravesar una etapa caracterizada por la formación de percepciones no realistas, durante la niñez tardía e inicios de la preadolescencia, los niños y niñas se muestran capaces de incorporar en su self las opiniones e imágenes que los otros tienen sobre ellos o ellas y, de esta forma, logran tener una visión más balanceada de su self. Este proceso se ve mediado por la experiencia escolar, en la cual pares, junto a padres y profesores, se convierten en otros significativos para el desarrollo del autoconcepto; los primeros sobretodo a través del rendimiento que muestran y las habilidades que poseen, y los segundos a través de las expectativas que tienen sobre ellos (Diehl, Lemerise, Caverly, Ramsay \& Roberts, 1998; Gipps \& Tusntall, 1998; Wentzel, 1998; Wentzel \& Wigfield, 1998). Todo este contexto social brindado por la escuela le permitirá al estudiante obtener información sobre su desempeño y diferenciar su motivación y capacidad en diferentes áreas académicas para formarse concepciones sobre sí mismo (Bouffard et al., 2003).

\section{Atribuciones causales de éxito y fracaso}

Las teorías de la atribución o explicaciones cognoscitivas de la motivación parten de la suposición de que todas las personas se 
cuestionan sobre la razón de sus éxitos y fracasos como un intento de comprender porqué sucedieron. De esta forma suelen atribuir sus éxitos y fracasos a la habilidad, esfuerzo, estado de ánimo, conocimiento, suerte, entre otros. Weiner (2000), el principal teórico de las atribuciones, postuló que los estudiantes atribuyen sus éxitos y fracasos principalmente a cuatro elementos: habilidad, esfuerzo, dificultad de la tarea y suerte, y las clasificó de acuerdo a tres dimensiones: locus de causalidad, estabilidad y controlabilidad. El locus de causalidad puede ser interno (e.g. habilidad, esfuerzo) o externo (e.g. azar, dificultad de la tarea). Asimismo, las causas escogidas pueden ser estables (e.g. la habilidad) o inestables (e.g. el esfuerzo, la suerte).

De otro lado, al igual que con el autoconcepto, la escuela también ofrece un ambiente importante para la formación de atribuciones. Diferentes estudios señalan que durante los primeros años de la escuela, los estudiantes dan mayor importancia al esfuerzo que a la habilidad (Gipps \& Tunstall, 1998). "Los niños pequeños creen que los alumnos trabajadores y dóciles tienen mayores probabilidades de alcanzar éxito y, por lo tanto, son más dignos de elogio y admiración" (Covington, 2000, p. 85). Años después la importancia del esfuerzo se debilita y se sustituye por la convicción de que la capacidad por sí sola basta para tener éxito, y esto se da porque los estudiantes comienzan a pensar que el elogio que los profesores dan por el esfuerzo que realizan es sinónimo de falta de capacidad y empiezan a ver la habilidad como requisito para el éxito (Covington, 2000). En este ambiente el logro se convierte en un determinante para la formación de atribuciones y el feedback de los profesores cobrará un rol importante (Thompson \& Hepburn, 2003). 


\section{Relaciones entre autoconcepto académico, atribuciones causa-} les de éxito y fracaso, y rendimiento académico

Como se ha podido apreciar, son numerosas las investigaciones que confirman las relaciones entre las atribuciones de éxito y fracaso y el autoconcepto académico con el rendimiento, pues crean un ambiente que puede predisponer al estudiante, ya sea positiva o negativamente, al aprendizaje y a implicarse o no en su proceso, Sin embargo, los resultados se tornan polémicos y faltos de consenso cuando se pretende establecer relaciones de causalidad entre dichas variables. Seguidamente, se presenta una revisión bibliográfica sobre las relaciones halladas entre estas tres variables tomadas de dos en dos para una mejor comprensión.

Sobre las atribuciones causales y el rendimiento académico, se tiene que muchos autores consideran a las atribuciones como las principales determinantes de la motivación de logro por su influencia en las expectativas, reacciones afectivas y rendimiento (Weiner, como se cita en González-Pienda, Núñez, González-Pumariega, Alvarez, Roces \& García, 2002). Si bien no se ha encontrado una relación de causalidad definitiva entre ambas variables, se puede identificar el papel que cumplen la habilidad y el esfuerzo en el rendimiento académico; así, el fracaso que se percibe fue causado por la falta de habilidad provocará un deterioro en el rendimiento, mientras que el fracaso atribuido a la falta de esfuerzo puede incrementar el rendimiento, ya que la persona puede tomar alguna medida para mejorar, por ejemplo, poner más esfuerzo (Gipps \& Tunstall, 1998).

Con relación al autoconcepto académico y al rendimiento académico, también resulta difícil determinar relaciones de causalidad entre estas dos variables, más bien se encuentran posturas que apoyan la existencia de una relación recíproca. Arancibia et al. (1990) señalan que si un estudiante tiene una imagen positiva 
de sí mismo, tendrá mayores posibilidades de éxito al enfrentarse a tareas que un estudiante que piensa que no es capaz. Del mismo modo, conforme un estudiante vaya logrando un buen desempeño académico, tendrá una imagen positiva de sí mismo y sostendrá posibilidades de éxito a futuro. Contextualizando estas relaciones por contenidos académicos, Marsh señala que el rendimiento matemático tiene un efecto positivo directo sobre el autoconcepto matemático, pero un efecto menos sustancial y negativo que sobre el autoconcepto en lectura. Por su parte, el rendindimiento en lectura tiene un efecto directo positivo y sustancial sobre el autoconcepto de lectura pero un efecto menos sustancial y negativo sobre el autoconcepto matemático (Marsh, 1984; Marsh, 1990; Marsh, Cairns, Relich, Barnes \& Debus, 1984).

Finalmente, sobre las relaciones entre el autoconcepto académico y las atribuciones causales de éxito y fracaso, son diversos los estudios que se han ocupado en determinar las diferencias que puede haber para la formación del autoconcepto, el hacer determinado tipo de atribuciones. Marsh et al. (1984) citan las investigaciones de Ickes y Layden, quienes encontraron que personas con alto autoconcepto mostraban mayor tendencia a atribuir su éxito a causas internas (e.g. habilidad) mientras que personas con bajo autoconcepto tendían a atribuir resultados positivos a causas externas (e.g. suerte). Frente a resultados negativos, las personas con alto autoconcepto o hacían atribuciones externas o señalaban todas las causas externas como improbables, mientras que las personas con bajo autoconcepto tendían a internalizar la responsabilidad por los resultados negativos. Esta acèptación de más responsabilidad por el éxito que por el fracaso es lo que Marsh denomina "hedonic bias" o self serving effect el cual reflejaría un intento por proteger la autoestima.

Finalmente, Marsh et al. (1984) analizaron las relaciones entre estas dos variables por contenidos específicos, hallando que las 
atribuciones a la habilidad en matemática correlacionan más con el autoconcepto matemático que con el autoconcepto lectura, y relaciones contrarias se encuentran para las atribuciones de habilidad en lectura.

La escuela provee un ambiente importante para la formación de autopercepciones en los estudiantes las cuales los llevarán a interpretar las experiencias que en ella viven.

Entre todas ellas, el rendimiento académico es el que resalta pues en él se centran muchos procesos relacionados con la formación de la imagen de sí mismo. Es en este contexto que se hace fundamental conocer las relaciones que se establecen entre el rendimiento académico y variables como el autoconcepto académico y las atribuciones causales de éxito y fracaso para comprender cómo éstas actúan en la autorregulación del comportamiento y del aprendizaje.

Por lo expuesto anteriormente las preguntas de investigación planteadas por el estudio fueron:

1. ¿Cuál es la relación entre el autoconcepto académico y las atribuciones causales de éxito y fracaso?

2. ¿Cuál es la relación entre el rendimiento académico y las atribuciones causales de éxito y fracaso?

3. ¿Cuál es la relación entre el autoconcepto académico y el rendimiento académico?

\section{Metodología}

La presente investigación corresponde con un estudio descriptivo correlacional ya que se aplicaron medidas sobre la muestra para luego encontrar las relaciones establecidas entre las variables. 
Las variables de estudio que se plantearon fueron:

- Autoconcepto académico: parte del autoconcepto que se relaciona directamente con el rendimiento académico ya sea en las áreas de matemática, comunicación integral y otras áreas académicas. Esta variable es medida con las escalas "autoconcepto matemático", "autoconcepto en lectura" y "académico general" del Cuestionario de Autodescripción (SDQ I).

- Atribuciones causales de éxito y fracaso: razones que da el alumno para explicar su éxito o fracaso académico en términos de esfuerzo, habilidad y/o causas externas. Esta variable es medida con la Escala de Atribuciones de Sydney (SAS).

- Rendimiento académico: las calificaciones obtenidas por los alumnos durante el año escolar en las áreas curriculares de matemáticas y comunicación integral.

\section{Participantes}

La muestra estuvo conformada por 284 estudiantes de ambos sexos pertenecientes al $5^{\circ}$ y $6^{\circ}$ grado de educación primaria de dos colegios estatales de Lima. La distribución por grado y sexo se aprecian en los siguientes cuadros.

\section{Cuadro 1}

Estadísticos descriptivos de la edad de la muestra según sexo

\begin{tabular}{lcrcc}
\hline & \multicolumn{4}{c}{ Edad } \\
\cline { 2 - 5 } & $M$ & $D E$ & Min. & Max. \\
\hline Masculino & 11.28 & 1.15 & 9 & 15 \\
Femenino & 11.02 & .88 & 9 & 14 \\
\hline
\end{tabular}


Relaciones entre autoconcepto académico, atribuciones de éxito y fracaso

Cuadro 2

Distribución de la muestra según grado escolar

\begin{tabular}{lccc}
\hline & \multicolumn{2}{c}{ Grado } & Total \\
\cline { 2 - 3 } & $5^{\circ}$ & $6^{\circ}$ & \\
\hline Masculino & 80 & 78 & 158 \\
Femenino & 75 & 54 & 129 \\
Total & 155 & 132 & 287 \\
\hline
\end{tabular}

\section{Instrumentos}

Cuestionario de Autodescripción - I (Self Description Questionnaire, $S D Q-1)$

Este instrumento fue elaborado por Herbert W. Marsh (1990) para evaluar el autoconcepto en dos dimensiones: académica y no académica. El instrumento está conformado por siete escalas que responden a las dimensiones señaladas: matemática, lectura y académico general para la dimensión académica, y las escalas de apariencia física, relación con padres, habilidad física y relación con pares para la dimensión no académica. Además se tiene una escala independiente a ambas dimensiones Ilamada "self general". Cada una de las escalas está conformada por ocho ítems los cuales hacen un total de 64 ítems. Finalmente se tienen escalas totales: "total académico", "total no académico" y "self total".

El SDQ-I emplea una escala ordinal de cinco puntos que son "falso", "casi siempre falso", "algunas veces falso, algunas veces verdadero", "casi siempre verdadero" y "verdadero", los cuales se califican con uno, dos, tres, cuatro y cinco puntos respectivamente. 
El instrumento fue construido con una muestra de 3562 niños australianos que cursaban estudios primarios en diferentes grados y reporta altos niveles de confiabilidad Alfa de Cronbach para cada una de las escalas tal como lo muestra el Cuadro 3.

\section{Cuadro 3}

Índice de confiabilidad por escala del SDQ-I para la muestra original $(N=3562)$

\begin{tabular}{lc}
\hline \multicolumn{1}{c}{ Escala } & Coeficiente Alfa \\
\hline Habilidad física & .83 \\
Apariencia física & .90 \\
Relación de pares & .85 \\
Relación con padres & .80 \\
Lectura & .89 \\
Matemáticas & .89 \\
Académica general & .86 \\
Self general* & .81 \\
Total no académico & .91 \\
Total académico & .92 \\
Total self & .94 \\
\hline$* n=739$ &
\end{tabular}

Escala de Atribuciones de Sydney (Sydney Attribution Scale, SAS)

También elaborado por H. W. Marsh (1984), este instrumento está formado por doce escalas que resultan de la combinación de tres factores: resultado (éxito y fracaso), contenido (matemática y lectura) y causas percibidas (esfuerzo, habilidad y causas externas). Cada escala tiene seis ítems los que hacen un total de 72 ítems.

El instrumento presenta 24 situaciones hipotéticas de éxito o fracaso en matemática o en lectura. En cada situación hipotética, el estudiante debe evaluar las posibles causas (habilidad, esfuerzo 
Relaciones entre autoconcepto académico, atribuciones de éxito y fracaso

y causas externas), calificando cada una de ellas con una escala ordinal de cinco puntos similar a la escala empleada en el SDQ.

La confiabilidad Alfa de Cronbach de cada una de las escalas reportada por la muestra original $(n=248)$ con la primera versión del instrumento (originalmente consta de 180 ítems repartidos en 18 escalas, las cuales incluyen el contenido "académica general" además de matemática y lectura) muestra índices que varían entre .57 y .90 . El Cuadro 4 muestra estos resultados.

\section{Cuadro 4}

Índice de confiabilidad por escala del SAS para la muestra original $(N=248)$

\begin{tabular}{lc}
\hline Escala & Coeficiente Alfa \\
\hline Matemática /éxito /habilidad (MSA) & .90 \\
Matemática /éxito /esfuerzo (MSE) & .76 \\
Matemática /éxito /causas externas (MSX) & .66 \\
Lectura /éxito /habilidad (RSA) & .92 \\
Lectura /éxito /esfuerzo (RSE) & .81 \\
Lectura /éxito /causas externas (RSX) & .80 \\
Académico general /éxito /habilidad (GSA) & .85 \\
Académico general /éxito /esfuerzo (GSE) & .84 \\
Académico general /éxito /causas externas (GSX) & .67 \\
Matemática /fracaso /habilidad (MFA) & .86 \\
Matemática /fracaso /esfuerzo (MFE) & .78 \\
Matemática /fracaso /causas externas (MFX) & .57 \\
Lectura /fracaso /habilidad (RFA) & .87 \\
Lectura /fracaso /esfuerzo (RFE) & .76 \\
Lectura /fracaso /causas externas (RFX) & .71 \\
Académíco general /fracaso /habilidad (GFA) & .84 \\
Académico general /fracaso /esfuerzo (GFE) & .73 \\
Académico general /fracaso /causas externas (GFX) & .66 \\
\hline
\end{tabular}

Las siglas entre paréntesis corresponden a la nomenclatura empleada por los autores para denominar a cada una de las escalas: contenido $(\mathrm{M}=$ mathematics/matemáticas, $\mathrm{R}=$ reading/lectu$\mathrm{ra}, \mathrm{G}=$ general/académico general), resultado ( $\mathrm{S}=$ success/éxito y $\mathrm{F}=$ failure/fracaso) y causa percibida ( $\mathrm{A}=$ ability/habilidad, $\mathrm{E}=$ effort/esfuerzo y $\mathrm{X}=$ external causes /causas extemas). 


\section{Rendimiento académico}

Esta variable fue medida a través de los promedios finales de las áreas curriculares de matemática y comunicación integral registrados al término del año escolar. Para el análisis, las calificaciones de los alumnos fueron estandarizadas por salon y convertidas a puntajes $Z$. La distribución de las calificaciones se presenta en el Cuadro 5.

\section{Cuadro 5}

Distribución de calificaciones

\begin{tabular}{lcccccccc}
\hline & \multicolumn{4}{c}{ Masculino } & \multicolumn{4}{c}{ Femenino } \\
\cline { 2 - 9 } & $M$ & $D E$ & Max. & Min. & $M$ & $D E$ & Max. & Min. \\
\hline Comunicación & 15 & .16 & 19 & 10 & 14.34 & .15 & 18 & 10 \\
Matemáticas & 14.53 & .18 & 19 & 10 & 14.14 & .18 & 19 & 8 \\
\hline
\end{tabular}

\section{Procedimiento}

Se realizó el proceso de doble traducción para el SDQ y el SAS del inglés al castellano y viceversa, ya que era la primera vez que se empleaban en nuestro medio. Por la misma razón, ambos instrumentos fueron aplicados a una muestra piloto conformada por 19 estudiantes de $5^{\circ}$ y $6^{\circ}$ grado de un colegio de condiciones similares a las de la muestra final. Los resultados de esta aplicación revelaron la necesidad de ajustar lingüísticamente algunos ítems para que fuesen entendidos por los alumnos. Paralelamente, se halló la validez de contenido consultando a ocho expertos en el tema, quienes para los dos cuestionarios debían identificar cada ítem con su respectiva escala. La gran mayoría de ítems obtuvieron altos índices de acuerdo para los dos instrumentos (el $84.4 \%$ del Cuestionario de Autodescripción y el $93 \%$ del Cuestionario de Atribuciones). Los ítems que no alcanzaron niveles 
aceptables fueron refraseados. Finalmente, las versiones finales del SDQ y el SAS fueron aplicadas a la muestra definitiva por dos psicólogas en cada uno de los salones seleccionados. Asimismo, se recogieron los promedios anuales de las áreas de $\mathrm{Ma}$ temática y Comunicación Integral de los estudiantes que conformaban la muestra para obtener el indicador de rendimiento académico.

Respecto al análisis estadístico de los instrumentos, se halló primero la validez de constructo y la confiabilidad de los instrumentos para luego determinar la normalidad de los datos. Este análisis permitió identificar que la mayoría de las escalas no se encontraban normalmente distribuidas por lo cual se decidió emplear estadísticas no paramétricas. En ese sentido la naturaleza del estudio exigía la búsqueda de correlaciones de Spearman a dos colas.

Validez y confiabilidad del Cuestionario de Autodescripción y de la Escala de Atribuciones en la muestra de Lima

El SDQ se mostró como un instrumento válido y confiable. El análisis factorial permitió identificar siete factores que explicaron el $53.4 \%$ de la varianza. Los factores obtenidos reflejaron una redistribución de ítems diferente a la versión original que considera ocho ítems por escala. Pese a esto, el coeficiente Alfa de Cronbach reportado para todo el instrumento fue .93 . Además los coeficientes de confiabilidad por escala hallados luego del análisis factorial también fueron altos, tal como lo muestra el Cuadro 6. 


\section{Cuadro 6}

Conformación de escalas $S D Q$ e índices de confiabilidad para la muestra de Lima

\begin{tabular}{lccc}
\hline $\begin{array}{c}\text { Escalas } \\
\text { SDQ }\end{array}$ & Código & $\begin{array}{c}N^{\circ} \\
\text { items }\end{array}$ & $\begin{array}{c}\text { Coeficiente } \\
\text { Alfa }\end{array}$ \\
\hline Autoconcepto en Matemáticas & SDQ1 & 11 & .90 \\
Autoconcepto en Lectura & SDQ2 & 10 & .88 \\
Apariencia física & SDQ3 & 8 & .85 \\
Relación con padres & SDQ4 & 8 & .84 \\
Habilidad física & SDQ5 & 8 & .80 \\
Relación con pares & SDQ6 & 8 & .73 \\
Escolaridad general & SDQ7 & 3 & .69 \\
\hline
\end{tabular}

Respecto a la Escala de Atribuciones ( $S A S$ ) se empleó el análisis factorial de segundo orden basado en las medias de las escalas, el cual permitió hallar cinco factores que explicaron el $74.6 \%$ de la varianza total. Se realizó un análisis factorial de segundo orden dado que el análisis de primer orden unía los ítems de las doce escalas en una forma teóricamente no coherente. El Cuadro 7 muestra la distribución de las nuevas escalas, el código asignado a cada una de ellas, el numero final de ítems y los coeficientes Alfa de Cronbach hallados para cada escala.

\section{Cuadro 7}

Conformación de escalas SAS e índices de confiabilidad para la muestra de Lima

\begin{tabular}{|c|c|c|c|}
\hline $\begin{array}{l}\text { Escalas } \\
\text { SAS }\end{array}$ & Código & $\begin{array}{l}N^{\circ} \\
\text { items }\end{array}$ & $\begin{array}{c}\text { Coeficiente } \\
\text { Alfa }\end{array}$ \\
\hline $\begin{array}{l}\text { Fracaso en matemáticas y lectura } \\
\text { por falta de habilidad y esfuerzo }\end{array}$ & SAS1 & 24 & .85 \\
\hline $\begin{array}{l}\text { Éxito en lectura por esfuerzo y } \\
\text { habilidad }\end{array}$ & SAS2 & 10 & .82 \\
\hline $\begin{array}{l}\text { Éxito en matemáticas y lectura } \\
\text { por causas externas }\end{array}$ & SAS3 & 12 & .74 \\
\hline $\begin{array}{l}\text { Fracaso en matemáticas y lectura } \\
\text { por causas externas } \\
\text { Éxito en matemáticas por habilidad }\end{array}$ & SAS4 & 12 & .66 \\
\hline y esfuerzo & SAS5 & 12 & .85 \\
\hline
\end{tabular}


Relaciones entre autoconcepto académico, atribuciones de éxito y fracaso

\section{Resultados}

Si bien el estudio se centra en el aspecto académico, se decidió incluir también las escalas no académicas del SDQ en el análisis estadístico con el fin de tener una visión más integral de los efectos que tienen sobre el rendimiento académico las diferentes dimensiones del autoconcepto.

El Cuadro 8 muestra las correlaciones entre las puntuaciones obtenidas en la escala de atribuciones de éxito y fracaso con las dimensiones académicas y no académicas del self. Se puede observar que las atribuciones al éxito en lectura y matemáticas, debido a causas internas, (SAS2 y SAS5) correlacionan positivamente con todas las dimensiones del autoconcepto. Asimismo, existe una alta correlación de dichas escalas con sus correspondientes autoconceptos académicos. De otro lado, las escalas de atribuciones externas (SAS3 y SAS4) fueron las que casi no presentaron correlaciones con las escalas de autoconcepto. Sólo se hallaron algunas excepciones de SAS4 con el autoconcepto matemático (SDQ1), de lectura (SDQ2) y de relación con pares (SDQ6), siendo todas ellas correlaciones negativas. Asimismo, se observaron relaciones negativas y altamente significativas entre las atribuciones SAS1 con las escalas de autoconcepto, principalmente las académicas (SDQ1, SDQ2 y SDQ7), y en menor grado con las escalas de apariencia física (SDQ3) y relación con pares (SDQ6).

Finalmente, la escala de atribuciones al éxito por causas externas (SAS3) no presentó correlaciones con ninguna de las dimensiones del autoconcepto. 


\section{Cuadro 8}

Correlaciones entre puntuaciones de autoconcepto $(S D Q)$ y atribuciones de éxito y fracaso (SAS)

\begin{tabular}{|c|c|c|c|c|c|}
\hline & $\begin{array}{c}\text { Fracaso } \\
\text { Mat/lect } \\
\text { Hab/esf } \\
\text { SAS1 }\end{array}$ & $\begin{array}{l}\text { Éxito } \\
\text { Lectura } \\
\text { Hab/esf } \\
\text { SAS2 }\end{array}$ & $\begin{array}{c}\text { Éxito } \\
\text { Mat/lect } \\
\text { C. externas } \\
\text { SAS3 }\end{array}$ & $\begin{array}{c}\text { Fracaso } \\
\text { Mat/lect } \\
\text { C. Exter. } \\
\text { SAS4 }\end{array}$ & $\begin{array}{c}\text { Éxito } \\
\text { Matemática } \\
\text { Hab/esf } \\
\text { SAS5 }\end{array}$ \\
\hline A. Matemático (SDQ1) & $-.41 * *$ & $.34 * *$ & -.10 & $-.20 * *$ & $.64 * *$ \\
\hline A. en Lectura (SDQ2) & $-.31 * *$ & $.59 * *$ & -.07 & $-.14 *$ & $.34^{* *}$ \\
\hline Apariencia física (SDQ3) & $-.15^{*}$ & $.30 * *$ & -.06 & -.01 & $.20^{* *}$ \\
\hline Relación con padres (SDQ4) & 4) -.11 & $.21^{* *}$ & .01 & -.08 & $.24^{* *}$ \\
\hline Habilidad física (SDQ5) & -.09 & $.18^{* *}$ & .05 & -.11 & $.18^{* *}$ \\
\hline Relación con pares (SDQ6) & $-.19 * *$ & $.28 * *$ & -.01 & $-.16^{*}$ & $.26^{* *}$ \\
\hline Académico general (SDQ7) & $-.29 * *$ & $.36^{* *}$ & -.08 & -.09 & $.50 * *$ \\
\hline
\end{tabular}

${ }^{*} \mathrm{p}>.05,{ }^{* *} \mathrm{p}>.01$

De otro lado, pueden identificarse relaciones de correspondencia entre las escalas de ambos instrumentos de acuerdo al área académica; así, la escala de atribuciones de éxito en matemáticas por habilidad y esfuerzo (SAS5) está altamente correlacionada con el autoconcepto matemático (SDQ1), en menor grado correlacionada con el autoconcepto académico general (SDQ7) y menos correlacionada con el autoconcepto en lectura (SDQ2) y las demás escalas de autoconcepto no académico. Relaciones similares se pueden encontrar con la escala de atribuciones al éxito en lectura por habilidad y esfuerzo (SAS2), la cual está más correlacionada con el autoconcepto en lectura (SDQ2), que con el autoconcepto académico general (SDQ7) y autoconcepto matemático (SDQ1) y en menor grado con las escalas de autoconcepto no académico.

Con respecto a las atribuciones causales y el rendimiento académico, se puede observar que las calificaciones en matemáticas y comunicación integral correlacionan significativamente con todas 
Relaciones entre autoconcepto académico, atribuciones de éxito y fracaso

las escalas de atribuciones, excepto con la escala de atribuciones de fracaso en matemáticas y lectura por causas externas (SAS4). Asimismo, debe señalarse que las correlaciones entre atribuciones causales y rendimiento fueron negativas cuando se trató de la escala de atribuciones de fracaso en matemáticas y lectura por falta de esfuerzo y habilidad (SAS1) y de la escala de atribuciones de éxito en matemática y lectura por causas externas (SAS3) (ver Cuadro 9).

\section{Cuadro 9}

Correlaciones entre rendimiento académico en matemáticas y comunicación integral, y las puntuaciones en la escala de atribuciones de éxito y fracaso

\begin{tabular}{lccccc}
\hline & Fracaso & Éxito & Éxito & Fracaso & Éxito \\
& Mat/lect & Lectura & Mat/lect & Mat/lect & Matemática \\
& Hab/esf & Hab/esf & C. externas & C. Exter. & Hab/esf \\
& SAS1 & SAS2 & SAS3 & SAS4 & SAS5 \\
\hline RCOM & $-.21^{* *}$ & $.28^{* *}$ & $-.13^{*}$ & .04 & $.35^{* *}$ \\
RMAT & $-.24^{* *}$ & $.22^{* *}$ & $-.27^{* *}$ & -.05 & $.35^{* *}$ \\
\hline
\end{tabular}

${ }^{*} \mathrm{p}>.05, * * \mathrm{p}>.01$

Al igual que en el caso anterior se halló relaciones de correspondencia por área académica entre el rendimiento académico y las escalas de atribución aunque éstas no fueron tan determinantes. Mientras el rendimiento en matemática correlacionó positivamente con las atribuciones al éxito en matemáticas por causas internas (SAS5) y menos con las atribuciones al éxito en lectura por causas internas (SAS2), el rendimiento en comunicación (RCOM) correlacionó más con las atribuciones al éxito en matemáticas por habilidad y esfuerzo (SAS5) que con su escala similar en lectura (SAS2).

Finalmente, sobre las relaciones entre el rendimiento académico y las escalas de autoconcepto, sólo se hallaron correlaciones 
altamente significativas cuando se trató de las tres escalas académicas del SDQ. No se hallaron correlaciones entre el rendimiento y las escalas no académicas, excepto la escala de habilidad física (SDQ5) la cual correlacionó negativamente con la nota de comunicación (RCOM). El Cuadro 10 muestra estos resultados.

\section{Cuadro 10}

Correlaciones entre rendimiento académico y autoconcepto académico y no académico

\begin{tabular}{lccccccc}
\hline & Matemática & Lectura & \multicolumn{3}{c}{ Apariencia } & \multicolumn{3}{c}{ Relación } & Habilidad Relación & Académico \\
& & & física & padres & física & padres & general \\
\hline RCOM & $.21^{* *}$ & $.15^{* *}$ & .08 & .11 & $-.16^{* *}$ & .00 & $.37^{* *}$ \\
RMAT & $.25^{* *}$ & .07 & .10 & .11 & -.10 & .00 & $.34^{* *}$ \\
\hline
\end{tabular}

${ }^{*} \mathrm{p}>.05,{ }^{* *} \mathrm{p}>.01$

También se pueden apreciar algunas relaciones de correspondencia entre estas dos variables; así se encuentra que el rendimiento en comunicación (RCOM) correlaciona más con el autoconcepto académico general (SDQ7), luego con el autoconcepto matemático (SDQ1) y en último lugar con el autoconcepto en lectura (SDQ2). Por su parte, el rendimiento en matemáticas (RMAT) correlaciona más con el autoconcepto académico general (SDQ7), luego con el autoconcepto en matemáticas (SDQ1) y no correlaciona con el autoconcepto en lectura (SDQ2).

En el Anexo se muestran de manera global las correlaciones entre todas las variables: autoconcepto, atribuciones de éxito y fracaso, ambas explicadas por sus respectivas escalas resultantes, y rendimiento académico. De esta forma, sobre las relaciones halladas entre las escalas SAS se encuentra que la escala de atribuciones causales de éxito en lectura y matemática por causas externas (SAS3) correlaciona positivamente con la escala de atribución de fracaso en lectura y matemática por falta de habilidad y esfuerzo 
(SAS1) y la escala de fracaso en lectura y matemática por causas externas (SAS4). También correlaciona negativamente con las atribuciones de éxito en lectura por habilidad y esfuerzo (SAS2). Por su parte, la escala de atribuciones de fracaso en lectura y matemáticas por causas externas (SAS4) correlaciona positivamente con las atribuciones al fracaso por falta de habilidad y esfuerzo (SAS1).

Con relación a las escalas de autoconcepto se observan correlaciones altamente significativas entre todas sus escalas, siendo éstas altas cuando se tratan de correlaciones entre escalas académicas (SDQ1, SDQ2 y SDQ7). Sobre las correlaciones entre escalas no académicas resalta la fuerte relación entre apariencia física (SDQ3) y relación de pares (SDQ6).

\section{Discusión}

El presente estudio se propuso ampliar la información sobre algunas de las variables psicológicas que guardan relación con el rendimiento académico en niños preadolescentes peruanos, siendo sus principales variables de estudio las atribuciones causales de éxito y fracaso y el autoconcepto académico. De esta forma se intenta tener una mayor comprensión del significado que tiene para los niños y niñas el estar en un ambiente escolar y también de la forma como explican sus logros y fracasos, todo esto con el fin de contribuir con los docentes en el logro del bienestar y desarrollo psicológico de los alumnos.

En relación a la validez y confiabilidad de los instrumentos empleados, el Cuestionario de Autodescripción (SDQ I) y la Escala de Atribuciones de Sydney (SAS), los índices hallados fueron altos. Respecto al Cuestionario de Autodescripción (SDQ), los siete factores obtenidos luego del análisis factorial reflejan en su 
estructura la conformación original concebida por el autor. El porcentaje de varianza explicada por estos factores $(53.4 \%)$ resulta bastante aceptable y por lo tanto refleja que el instrumento puede brindar información válida sobre el autoconcepto del niño en estas siete áreas.

Cabe señalar que el análisis factorial estableció un ordenamiento de ítems ligeramente diferente al cuestionario original, sin que esto signifique una alteración en la estructura teórica del instrumento. Por el contrario, se pudo ratificar la propuesta de Marsh, Harter y otros autores quienes señalan que los niños o preadolescentes son capaces de distinguir entre las diferentes dimensiones de su self y establecer diferentes percepciones para cada una de ellas (Bong \& Skaalvik, 2003; Harter, 1990; Hattie, 1992; Marsh, 1990).

Sobre la confiabilidad del SDQ, el índice reportado fue muy alto (coeficiente Alfa de Cronbach $=.93$ ), lo cual indica la alta consistencia interna del instrumento. Todos los ítems correlacionaron positivamente con el total de la prueba, a la par que mostraron capacidad de discriminación en las respuestas dadas por los alumnos. Los coeficientes Alfa de Cronbach hallados para cada escala, la mayoría entre .90 y .69 , también reflejan su alta o aceptable consistencia y por ende su capacidad para medir las siete escalas del autoconcepto.

De otro lado, los resultados de validez y confiabilidad para la Escala de Atribuciones de Sydney (SAS) también fueron positivos. El análisis factorial de segundo orden aplicado para hallar la validez identificó cinco escalas que explican el $74.57 \%$ de la varianza, porcentaje altamente positivo y que asegura la variabilidad de las respuestas en estos cinco factores. 
Sobre la confiabilidad del SAS, el coeficiente Alfa reportado fue alto (.80) mostrando así su consistencia interna. Además, la formación de las nuevas escalas luego del análisis factorial secundario mejoró los coeficientes Alfa obtenidos para las doce escalas originales.

Las escalas conformadas ratifican algunas de las propuestas que sus autores consideraron para su elaboración, como por ejemplo la diferenciación de atribuciones causales por resultado (éxito y fracaso), pero también se hallaron diferencias. En ese sentido, un elemento que contrasta con los factores hallados por Marsh et al. (1984) radica en la unión de la habilidad y esfuerzo como causas percibidas de éxito o fracaso en un sólo factor, tal como se observa para los casos de las escalas SAS1 (atribuciones causales al fracaso en matemáticas y lectura por falta de habilidad y esfuerzo), SAS2 (atribuciones causales al éxito en lectura por habilidad y esfuerzo) y SAS5 (atribuciones causales al éxito en matemáticas por habilidad y esfuerzo). Esta estructura podría indicar que los niños de la muestra manifiestan un alto locus de control interno y no diferencian aún entre ambas causas, habilidad y esfuerzo, cuando están en situaciones de logro. Esto difiere de lo reportado en diversas investigaciones que señalan que los niños de esta edad son capaces de distinguir entre el esfuerzo y la habilidad, valorando inclusive más a la habilidad que al esfuerzo (Covington, 2000; Gipps \& Tunstall, 1998; Marsh, 1984).

Una explicación a estos resultados podría ser que los alumnos de la muestra aun siguen dando un alto valor a la retroinformación que reciben de sus profesores sobre el esfuerzo que ponen en sus tareas y por ello lo consideran tan importante como la habilidad. Otra causa podría ser que la retroalimentación que los estudiantes reciben de sus profesores corresponde con una retroalimentación de tipo no contingente, es decir, no es lo suficientemente clara y no les permite identificar si sus logros se deben al esfuerzo o a la habilidad (Thompson \& Hepburn, 2003). 
Cabe señalar que entre los objetivos iniciales de este estudio no se encontraba la evaluación del ambiente escolar y sus influencias en la motivación y autoconcepto del alumno, sin embargo, estos puntos de discusión resultan válidos. Sería necesario entonces recoger más información sobre el entorno familiar y escolar en el que se forman los niños y niñas para encontrar una explicación de por qué los participantes de la muestra ven al esfuerzo y la habilidad en el mismo nivel.

Asimismo, debe precisarse que la unión de ambas causas percibidas limita las posibilidades del estudio pues no permite apreciar la preferencia del alumno por atribuir el fracaso a la falta de esfuerzo antes que a la falta de habilidad. Sin embargo, es importante analizar las implicancias que tiene para el niño ver a la habilidad y al esfuerzo al mismo nivel cuando se enfrenta al fracaso (representado en la escala SAS1). Este hecho significa una alta internalización de responsabilidad que podría tener consecuencias negativas en el autoconcepto. El poner estas causas internas en una misma categoría elimina la opción de atribuir el fracaso a no haberse esforzado lo suficiente y, por lo tanto, reduce su repertorio de posibilidades de formas de proteger su autovalía a las causas externas, lo que podría llevar al alumno a una situación de mayor fragilidad (Thompson \& Dinnel, 2003).

En conclusión, es posible encontrar explicaciones a la unión de esfuerzo y habilidad en un sólo factor, sin embargo, se cree necesario profundizar en el estudio de la Escala de Atribuciones de Sydney. Su compleja estructura requiere de un análisis factorial confirmatorio mediante el programa estadístico LISREL tal cual lo sugieren sus autores $(\mathrm{H}$. W. Marsh, comunicación personal electrónica, 29 de agosto, 2003).

Asimismo, los factores hallados confirman las diferencias que pueden haber en el estilo atribucional según contenido académico, 
aunque esto sólo se pudo comprobar para las escalas de éxito por habilidad y esfuerzo, las cuales diferenciaron entre lectura y matemáticas (SAS2 y SAS5, respectivamente). Se podría deducir entonces que los niños de esta edad perciben que tener éxito en lectura es diferente a tener éxito en matemáticas cuando tienen las capacidades suficientes y ponen el esfuerzo necesario. Es conocido que la escuela enfatiza el desarrollo de capacidades matemáticas y de comunicación en los niños y, por lo tanto, invierte la mayor parte del tiempo de clases en la enseñanza de ambas áreas académicas. Sin embargo, existen indicios para afirmar que entre las dos áreas, la matemática es considerada más importante, la que es más objeto de diversos estereotipos y la que más reconocimiento trae de profesores y padres de familia.

De otro lado, la estructura factorial hallada no identificó factores conteniendo escalas de éxito y fracaso a la vez; excepto por el caso de la escala de fracaso en lectura por falta de habilidad (RFA), la cual mostró mayor carga para el factor que agrupaba escalas de éxito en lectura por habilidad y esfuerzo (SAS2). La pertenencia de la escala RFA a un factor más adecuado que reuniera a otras escalas de fracaso se debió no sólo a la carga factorial significativa que también mostraba con este componente, sino también a que mostraba mayor concordancia con los factores hallados por Marsh (1984).

Una última observación sobre la composición de factores del SAS se relaciona con las escalas de atribución a causas externas, las cuales unieron contenido académico y diferenciaron tipo de resultado. Así se tuvieron: SAS3 (atribuciones al éxito en lectura y matemáticas por causas externas) y SAS4 (atribuciones al fracaso en lectura y matemáticas por causas externas). Esto refleja la capacidad de los niños para discriminar entre el éxito obtenido por factores externos como la suerte y el azar y el fracaso «provocado» por las mismas razones. De esta forma, un niño o niña podría 
acudir a causas externas para explicar sus resultados cada vez que enfrente situaciones que reflejen bajo rendimiento como recurso para proteger su autoestima (Covington, 2000; Marsh et al., 1984). Asimismo, la identificación de una escala de atribuciones al éxito por causas externas debería plantear una alerta a los profesores sobre la posibilidad de que éste se constituya en un estilo atribucional en el alumno. Si un niño percibe que ningún elemento interno influye en los resultados que obtiene puede crearse un sentimiento de incapacidad, el cual puede agravarse y constituirse finalmente en situaciones de desesperanza aprendida (Gipps \& Tunstall, 1998).

En conclusión, de lo hipotetizado por Marsh et al. (1984) sobre características de un instrumento que mide atribuciones, sólo se halló una clara diferenciación de las atribuciones por resultado (éxito y fracaso) mas no por contenido académico (matemáticas y lectura) ni por causa percibida (habilidad, esfuerzo y causas externas) puesto que éstas dos últimas sólo se dieron parcialmente.

Sobre las relaciones entre el autoconcepto y las atribuciones causales de éxito y fracaso, se observaron diferentes resultados dependiendo del tipo de atribución. De este modo, se encontraron correlaciones positivas y altamente significativas entre las escalas de autoconcepto académico (matemático, lectura, académico general y total académico) con las atribuciones al éxito por esfuerzo y habilidad en las áreas de lectura y matemáticas (SAS2 y SAS5 respectivamente). Esto señalaría que un alumno que reconoce su esfuerzo y habilidad como causantes del éxito mostrará percepciones positivas sobre su desempeño en estas áreas académicas.

Cabe señalar que estas relaciones positivas se extienden aun para las dimensiones no académicas del self como apariencia física, relación con padres, habilidad física, relación con pares y self general, lo cual habla de la satisfacción y bienestar general que 
produce en los estudiantes el saber que los éxitos logrados se deben a su habilidad y esfuerzo.

De otro lado, las correlaciones negativas entre las atribuciones al fracaso por falta de esfuerzo y habilidad (SAS1) con las dimensiones académicas del autoconcepto estarían indicando que este tipo de atribuciones disminuye la percepción de capacidad que el alumno tiene de sí mismo al aceptar que no posee las condiciones para lograr éxito. Al mismo tiempo, este resultado señalaría que alumnos con altos niveles de autoconcepto académico mostrarían menor tendencia a hacer este tipo de atribuciones. De iste modo resultaría esperable que ante el fracaso las personas con alto autoconcepto busquen explicaciones relacionadas con el azar como una forma de protección a sí mismos.

Para respaldar esta lógica las correlaciones entre los autoconceptos matemático y de lectura con las atribuciones al fracaso por causas externas (SAS4) deberían ser positivas. Sin embargo los resultados mostraron correlaciones negativas.

Este hecho plantea la pregunta, si los alumnos con alto autoconcepto académico no atribuyen su fracaso a la falta de esfuerzo o habilidad y tampoco a causas externas, entonces ¿cómo explican el fracaso? La respuesta podría estar en los altos niveles de autoconcepto mostrados por los participantes, los cuales como Marsh señala, los lleva a negar toda posibilidad de fracaso. La ausencia de correlaciones entre las atribuciones de éxito a causas externas (SAS3) con el autoconcepto en sus dimensiones académicas estaría reforzando estos hallazgos.

De otro lado, las correlaciones negativas entre las escalas de fracaso por causas internas (SAS1) con las escalas no académicas como apariencia física y relación con pares deja entrever la interacción que tiene esțe tipo de atribuciones con las dimensiones no 
académicas del autoconcepto. Como se sabe, la apariencia física y la relación con pares se encuentran vinculadas con la aceptación por los demás, por lo que este resultado significaría que cuanto mayor sea el sentimiento que se tiene de ser acogido por los demás, menor será la tendencia a atribuir el fracaso a causas internas.

Otro resultado importante es la relativa ausencia de correlaciones de las escalas de atribuciones de éxito y fracaso por causas externas (representados por SAS3 y SAS4) con las diferentes escalas del autoconcepto. De este modo, se encuentra que este tipo de atribuciones no provoca mayores efectos en el autoconcepto, lo cual se relaciona con la tendencia de los participantes de no considerar las causas externas para explicar sus éxitos o fracasos.

Con relación al tipo de relaciones establecidas entre las atribuciones causales y el rendimiento académico, se hallaron correlaciones positivas y significativas cuando se trataron de atribuciones al éxito por causas internas (representados por SAS2 y SAS5), tal como se puede ver en el Cuadro 9. Esto significaría que cuanto más positivo sea el rendimiento que tenga un alumno más reconocerá a su esfuerzo y habilidad como causantes de este resultado.

Otra observación que puede hacerse de estos resultados se relaciona con la hipótesis de Marsh sobre las correspondencias que deben darse entre las atribuciones y el rendimiento según contenido académico (matemáticas y lectura). El Cuadro 9 permite apreciar que éstas se dieron parcialmente. Como resulta lógico, la correlación entre las atribuciones al éxito en lectura por causas internas (SAS2) y el rendimiento en comunicación fue mayor que la correlación de este tipo de atribuciones con el rendimiento en matemáticas. Sin embargo, no hubo tal diferenciación para el caso de matemáticas. Las correlaciones entre las atribuciones al éxito 
en matemáticas por causas internas (SAS5) y el rendimiento en matemáticas fue igual que con el rendimiento en comunicación.

Estos resultados hablarían del predominio de las matemáticas para formar atribuciones de éxito a causas internas. Asimismo, la motivación y expectativas que se tenga con esta área llevaría a obtener mejor rendimiento en las otras áreas. Así, queda confirmada la creencia de que un alumno o alumna que muestra un buen desempeño en matemáticas es más inteligente y por lo tanto tiene más probabilidades de éxito para todos los cursos.

De otro lado, la correlación altamente significativa y negativa entre las atribuciones al fracaso por causas internas (SAS1) con el rendimiento académico de matemáticas y comunicación muestra claramente que este estilo atribucional repercute negativamente sobre el rendimiento de un alumno en estas áreas. También indicaría que cuanto mayor sea el rendimiento en estas áreas académicas menos se atribuirá el fracaso a causas internas, buscando por el contrario causas externas para proteger su autoestima. Sin embargo, no se hallaron correlaciones entre rendimiento y atribuciones de fracaso a causas externas (SAS4). Estos resultados mantienen un patrón similar al explicado anteriormente entre el autoconcepto académico y las atribuciones del fracaso a causas externas. De esta forma se reafirma la tendencia entre los participantes a no atribuir el fracaso a causas externas y menos aún a causas internas, lo que, como lo señalaron Ickes y Layden (Marsh et al., 1984), resulta característico de niños con elevado autoconcepto.

Sobre las relaciones entre el autoconcepto y el rendimiento académico, éstas se manifestaron casi en su totalidad para las escalas académicas tal como se puede ver en el Cuadro 10.

Las correlaciones del rendimiento en matemáticas y comunicación y el autoconcepto académico general y el self académico 
hablan del peso que tiene un buen rendimiento en ambos cursos sobre el resto de materias, creando una especie de efecto de halo. Esto se podría deber, como se dijo anteriormente, a la importancia concedida a ambas áreas durante la escuela primaria.

Con respecto al rendimiento y las dimensiones no académicas del self, la casi inexistencia de relaciones entre estas dos variables confirmaría la capacidad de los participantes de la muestra para diferenciar las dimensiones de su self e identificar las que tienen relación directa con sus actividades escolares.

Por último, las relaciones de correspondencia entre el rendimiento y el autoconcepto según contenido académico no concuerdan con lo propuesto por Marsh. El rendimiento matemático correlaciona más con la escala académico general (SDQ7) y en segundo lugar correlaciona con el autoconcepto matemático, mas no con el autoconcepto en lectura. Se esperaría resultados similares para el caso del rendimiento en comunicación pero esta situación no se dio. La ausencia del tipo de relación esperada por Marsh para el área de lectura, más allá de la preeminencia de la escala académico general, se podría deber, una vez más, al uso del promedio final en el área de comunicación en vez de los resultados de una prueba de lectura ya que, como se sabe, la nota de comunicación incluye no sólo la evaluación de la capacidad lectora sino también de producción de textos y comunicación oral, y esto pudo provocar un desfase porque los instrumentos usados hacen alusión a la lectura.

\section{Conclusiones}

$\mathrm{Al}$ igual que en otras investigaciones, el autoconcepto y las atribuciones causales se mostraron fuertemente correlacionadas con el rendimiento académico. Si bien ambos son constructos 
diferentes, los estudiantes peruanos parecen integrarlos coherentemente para elaborar explicaciones de sus resultados académicos así como imágenes de su propia capacidad. De esta forma, si bien los estudiantes podían identificar las diferentes dimensiones de su autoconcepto, todas éstas, y no sólo las académicas, contribuían con la formación de atribuciones que les permiten proteger la propia valía.

De otro lado, los resultados permitieron evidenciar las influencias del ambiente y, en forma especial, del docente en la formación del autoconcepto y de las atribuciones causales, lo cual corrobora la dimensión social que subyace al autoconcepto. En ese sentido, se hace necesario recoger también información sobre cl docente para así determinar con mayor precisión las acciones que debe desplegar en el aula para favorecer la creación de percepciones positivas en sus alumnos. Del mismo modo, amerita la exploración de variables relacionadas con los padres quienes también influyen con sus expectativas, estereotipos y prejuicios, y también con los pares pues son el referente con quienes se miden. De todo esto se desprende la importancia de incidir en las investigaciones de variables de tipo motivacional no sólo con miras a mejorar el rendimiento de los estudiantes, sino sobre todo a lograr infundir en ellos un sentido de bienestar.

\section{Referencias}

Arancibia, V., Maltes, S. \& Alvarez, M. I. (1990). Test de Autoconcepto Académico. Estandarización para escolares de $1^{\circ}$ a $4^{\circ}$ año de enseñanza básica. Santiago: Universidad Católica de Chile.

Bong, M. \& Skaalvik, E. (2003). Academic self concept and self efficacy. How different are they really? Educational Psychology Review, 15(1), 1-40. 
Bouffard, T., Marcoux, M., Vezeau, C. \& Bordeleau, L. (2003). Changes in self-perceptions of competence and intrinsic motivation among elementary schoolchildren. British Journal of Educational Psychology, 73, 171-186. Recuperado el 11 de julio del 2005 de la base de datos ABI/INFORM.

Covington, M. (2000). La voluntad de aprender. Guía para la motivación en el aula. Madrid: Alianza.

Diehl, D. S., Lemerise, E. A., Caverly, S. L., Ramsay, S. \& Roberts, J. (1998). Peer relations and school adjustment in undergraded primary children. Journal of Educational Psychology, 90(3), 506-515.

Gipps, C. \& Tunstall, P. (1998). Effort, ability and the teacher: Young children's explanations for success and failure. Oxford Review of Education, 24(2), 149-165. Recuperado el 11 de julio del 2005 de la base de datos ABI/INFORM.

González-Pienda, J. A., Núñez, J. C., González-Pumariega, S., Alvarez, L., Roces, C. \& García, M. (2002). A structural equation model of parental involvement, motivational and aptitudinal characteristics, and academic achievement. The Journal of Experimental Education, 70(3), 257. Recuperado el 11 de Julio del 2005 de la base de datos ABI/INFORM.

Graham, S. (1991). A review of attribution theory in achievement contexts. Educational Psychology Review, 3(1), 5-39.

Harter, S. (1982). The perceived competence scale for children. Child Development, 53, 87-97.

Harter, S. (1999). The construction of the self. A developmental perspective. Nueva York: Guilford Press.

Hattie, J. (1992). Self-concept. Nueva Jersey: Lawrence Erlbaum.

Marsh, H. W. (1984). Relations among dimensions of self attributions, dimensions of self concept, and academic achievement. Journal of Educational Psychology, 76(6), 1291-1308.

Marsh, H. W. (1990). Self Description Questionnaire - I (SDQ-I) Manual. MacArthur, Australia: University of Western Sydney. 
Marsh, H. W., Cairns, L., Relich, J., Barnes, J. \& Debus, R. (1984). The relationship between dimensions of self concept. Journal of Educational Psychology, 76(1), 3-32.

Saura, P. (1995). La educación del autoconcepto: cuestiones y propuestas. Murcia: Universidad de Murcia.

Thompson, T. \& Dinnel, D. (2003). Construction and initial validation of the Self-Worth Protection Scale. British Journal of Educational Psychology, 73, 89. Recuperado el 11 de Julio del 2005 de la base de datos ABI/INFORM.

Thompson, T. \& Hepburn, J. (2003). Causal uncertainty, claimed and behavioural self handicapping. British Journal of Educational Psychology, 73, 274-266. Recuperado el 11 de Julio del 2005 de la base de datos ABI/INFORM.

Villaroel, V. (2001). Relación entre autoconcepto y rendimiento académico. Psykhe, 10(1), 3-18.

Weiner, B. (2000). Intrapersonal and interpersonal theories of motivation from an attributional perspective. Educational Psychology Review, 12(1), 1-14.

Wentzel, K. R. (1998). Social relationship and motivation in middle school. The role of parents, teachers, and peers. Journal of Educational Psychology, 90(2), 200-209.

Wentzel, K. R. \& Wigfield, A. (1998). Academic and social motivational influences on students' academic performance. Educational Psychology Review, 10(2), 155-175. 
Correlaciones entre atribuciones causales de éxito y fracaso, autoconcepto académico y rendimiento académico para la muestra total $(n=287)$

\begin{tabular}{|c|c|c|c|c|c|c|c|c|c|c|c|c|c|c|c|c|c|}
\hline & 1 & 2 & 3 & 4 & 5 & 6 & 7 & 8 & 9 & 10 & 11 & 12 & 13 & 14 & 15 & 16 & 17 \\
\hline 1 sas1 & 1 & & & & & & & & & & & & & & & & \\
\hline 2 sas 2 & $-.38 * *$ & 1 & & & & & & & & & & & & & & & \\
\hline 3 sas 3 & $.30 * *$ & $-.16^{*}$ & 1 & & & & & & & & & & & & . & & \\
\hline 4 sas 4 & $.43^{* *}$ & -.10 & $.31^{* *}$ & 1 & & & & & & & & & & & & & \\
\hline 5 sas 5 & $-.32 * *$ & $.52^{* *}$ & -.11 & -.11 & 1 & & & & & & & & & & & & \\
\hline $6 \mathrm{sdq} \mathrm{l}$ & $-.41 * *$ & $.34^{* * *}$ & -.10 & $-.20 * *$ & $.64^{* *}$ & 1 & & & & & & & & & & & \\
\hline 7 sdq2 & $-.31 * *$ & $.59 * *$ & -.07 & $-.14^{*}$ & $.34^{* *}$ & $.42 * *$ & 1 & & & & & & & & & & \\
\hline $8 \mathrm{sdq} 3$ & $-.15^{*}$ & $.30 * *$ & -.06 & -.01 & $.20^{* *}$ & $.30 * *$ & $.21^{* *}$ & 1 & & & & & & & & & \\
\hline 9 sdq4 & -.11 & $.21^{* *}$ & .01 & .08 & $.24 * *$ & $.38 * *$ & $.28 * *$ & $.25^{* *}$ & 1 & & & & & & & & \\
\hline $10 \mathrm{sdq} 5$ & .09 & $.18 * *$ & .05 & -.11 & $.18^{* *}$ & $.27 * *$ & $.19^{* *}$ & $.24^{* *}$ & $.26 * *$ & 1 & & & & & & & \\
\hline 11 sdq6 & $-.19 * *$ & $.28 * *$ & -.01 & $-.16^{*}$ & $.26 * *$ & $.31^{* *}$ & $.31^{* *}$ & $.38^{* *}$ & $.26^{* *}$ & $.32^{* *}$ & 1 & & & & & & \\
\hline $12 \mathrm{sdq} 7$ & $-.29 * *$ & $.36 * *$ & -.08 & -.09 & $.50^{* *}$ & $.51^{* *}$ & $.41^{* *}$ & $.28 * *$ & $.37 * *$ & .12 & $.24^{* *}$ & 1 & & & & & \\
\hline 13 Tot Acad. & $-.40 * *$ & $.50 * *$ & -.08 & $-.18 * *$ & $.61^{* *}$ & $.81^{* *}$ & $.72^{* *}$ & $.32 * *$ & $.44^{* *}$ & $.23 * *$ & $.33^{* *}$ & $.82^{* *}$ & 1 & & & & \\
\hline 14 Tot No acad. & $-.13^{*}$ & $.32 * *$ & .03 & -.08 & $.31^{* *}$ & $.42 * *$ & $.33^{* *}$ & $.72^{* *}$ & $.59 * *$ & .62 & $.68 * *$ & $.36 * *$ & $.45^{* *}$ & 1 & & & \\
\hline 15 Total Self & $-.33^{* *}$ & $.48 * *$ &. .02 & $-.16^{*}$ & $.55^{* *}$ & $.75^{* *}$ & $.63^{* *}$ & $.56^{* *}$ & $.59 * *$ & $.45^{* *}$ & $.57^{* *}$ & $.72^{* *}$ & $.88^{* *}$ & $.80^{* *}$ & 1 & & \\
\hline $16 \mathrm{RCOM}$ & $-.21^{* *}$ & $.28 * *$ & $-.13^{*}$ & .04 & $.35 * *$ & $.21^{* *}$ & $.15^{* *}$ & .08 & .11 & $-.16^{* *}$ & .00 & $.37^{* *}$ & $.31^{* *}$ & .01 & $.20^{* *}$ & 1 & \\
\hline 17 RMAT & $-.24^{* *}$ & $.22 * *$ & $-.27 * *$ & -.05 & $.35^{* *}$ & $.25 * *$ & .07 & .10 & .11 & -.10 & .00 & $.34^{* *}$ & $.28 * *$ & .05 & $.20 * *$ & $.76^{* *}$ & 1 \\
\hline
\end{tabular}

